\title{
DESEMPENHO SILVICULTURAL DE CLONES DE Eucalyptus grandis EM RELAÇÃo Às ÁRVORES MATRIZES ${ }^{1}$
}

\author{
Glêison Augusto dos Santos², Aloisio Xavier ${ }^{3}$ e Hélio Garcia Leite ${ }^{3}$
}

\begin{abstract}
RESUMO - Objetivando avaliar a eficiência da seleção inicial de 13 árvores-matriz para clonagem de Eucalyptus grandis e o desempenho delas em teste clonal, foram mensuradas as características dap, altura total $(H t)$, volume com casca $\left(\mathrm{V}_{\mathrm{CC}}\right)$, sem casca $\left(\mathrm{V}_{\mathrm{SC}}\right)$ e volume de casca $\left(\mathrm{V}_{\mathrm{C}}\right)$, bem como as cinco árvores "co-dominantes" em um raio de $10 \mathrm{~m}$ da matriz selecionada, para verificar a sua superioridade em relação aos seus pares. $\mathrm{O}$ desempenho silvicultural dos clones dessas matrizes foi avaliado aos sete anos de idade, em teste clonal instalado no delineamento em blocos ao acaso, com três repetições e parcela quadrada de 25 plantas, em espaçamento de 3 x $2 \mathrm{~m}$. Com base nos resultados deste trabalho, pode-se concluir que a avaliação da superioridade em $H t$ da árvore-matriz em relação às árvores co-dominantes do local de seleção é um parâmetro importante para a escolha inicial de árvores-matriz. A análise multivariada foi eficaz para a alocação das matrizes em grupos divergentes, bem como para a classificação de árvores-matriz selecionadas posteriormente dentro dos grupos pré-definidos pelo método de Tocher. Existe relação significativa entre as características utilizadas para a seleção inicial das árvores-matriz e o desempenho dos clones dessas matrizes na avaliação final do teste clonal. Esses resultados possibilitam a redução do número de material genético a ser avaliado. No entanto, a instalação dos testes é imprescindível no processo de seleção, principalmente devido à existência da interação do genótipo com os efeitos ambientais.
\end{abstract}

Palavras-chave: Melhoramento florestal, seleção de clones, teste clonal, análise multivariada e silvicultura clonal.

\section{SILVICULTURAL PERFORMANCE OF Eucalyptus grandis CLONES COMPARED WITH DONOR TREES}

\begin{abstract}
Aiming at evaluating the efficiency of initial selection of thirteen donor trees for Eucalyptus grandis cloning and their performance in a clonal test, the characteristics diameter (dap), total height (Ht), volume with bark, $\left(V_{C C}\right)$, volume without bark $\left(V_{S C}\right)$ and bark volume $\left(V_{C}\right)$ were assessed along with five "codominant" trees within a $10 \mathrm{~m}$ radius of the selected donor, to evaluate its superiority compared to their pairs. The silvicultural performance of these clones was evaluated at seven years of age in a clonal test arranged in randomized block design with three repetitions and a square plot of 25 plants, in a 3 X 2 spacing. Results showed that the superior Ht of donor tree compared to the "co-dominant" trees of the selection site is an important parameter for the initial selection of donor tress. Multivariate analyzes was efficient for placing donor plants in divergent groups, as well as for the classification of donor trees selected afterwards, within the groups defined by the Tocher method. There is a significant relationship among the characteristics used for the initial selection of donor trees and the performances of their clones in the final evaluation of the clonal test. These results allow the reduction in the number of genetic material to be evaluated, however, the tests are essential for the selection process, mainly because the genotype-environment interaction effects.
\end{abstract}

Keywords: Forest improvement, clone selection, clonal test, multivariate analyses and clonal forestry.

\footnotetext{
${ }^{1}$ Recebido em 26.10.2004 e aceito para publicação em 05.04.2006.

${ }^{2}$ KLABIN S.A. (SC).

${ }^{3}$ Departamento de Engenharia Florestal da Universidade Federal de Viçosa, Viçosa-MG. E-mail: <xavier@ ufv.br>.
} 


\section{INTRODUÇÃO}

A silvicultura clonal de Eucalyptus por meio da seleção e propagação vegetativa de genótipos selecionados tem permitido o estabelecimento de florestas clonais, proporcionando maior uniformidade da matéria-prima florestal, melhor adaptação dos clones aos diferentes ambientes de plantio, maior produção de madeira por unidade de área, racionalização das atividades operacionais e redução na idade de corte (CAMPINHOS Jr. e IKEMORI, 1987; FERREIRA, 1992; REZENDE et al., 1994; SILVA, 2001; XAVIER, 2003).

Geralmente, têm-se adotado duas etapas na seleção e avaliação de clones de Eucalyptus. A primeira ocorre com grande número de clones, em que se adotam parcelas pequenas e em vários locais, utilizando a seleção precoce na identificação de clones superiores, com base em características silviculturais e tecnológicas da madeira. Na segunda etapa, os clones selecionados na etapa inicial são avaliados em experimentos maiores (escala-piloto), visando avaliar a "performance" representativa em plantio comercial. Essa estratégia tem fornecido bons resultados na área florestal, e sido recomendada como adequada em trabalhos recentes desenvolvidos com dados de campo de empresas florestais (SILVA, 2001; ANDRADE, 2002).

A seleção inicial das matrizes para a clonagem vem, contudo, sendo realizada através da observação direta de características dendrométricas (dap, Ht e volume) e de outras características fenotípicas desejáveis, como a forma do fuste, o tipo de casca, o tamanho da copa e a espessura e persistência dos ramos, além da presença de pragas e doenças. Essa metodologia levou as empresas florestais a um contraste entre o grande número de árvores selecionadas para a clonagem e o pequeno número de clones indicados para o plantio comercial, após as etapas de seleção (IPEF, 1994).

A baixa eficiência no processo de seleção inicial de árvores-matriz acarreta aumento no custo da silvicultura clonal, uma vez que a instalação de testes clonais com as matrizes selecionadas é imprescindível para que se possam selecionar efetivamente os melhores clones (KIKUTI, 1988; FRAMPTON Jr. e FOSTER, 1993; LAMBETH et al., 1994).

Barreiros et al. (2002), estudando a utilização da programação linear para a seleção de clones em teste clonais, relataram que a maior dificuldade encontrada, foi a carência de estudos que pudessem fornecer informações prévias mais detalhadas acerca da seleção de árvores-matriz.

Diante do exposto, observa-se a necessidade de estudos que permitam maior acurácia na seleção inicial da árvore-matriz para clonagem, correlacionando características utilizadas na seleção inicial das matrizes com o desempenho futuro delas após a avaliação definitiva em testes clonais e ou plantios comerciais.

Nesse sentido, o presente trabalhou objetivou realizar uma abordagem sobre a utilização de técnicas de análise multivariada na seleção inicial de árvores-matriz para clonagem de Eucalyptus grandis e a avaliação do desempenho dos clones dessas matrizes em teste clonal.

\section{MATERIAL E MÉTODOS}

\subsection{Material experimental}

A presente pesquisa foi realizada na Inpacel Agroflorestal, empresa pertencente ao Grupo International Paper, no Município de Arapoti, Paraná (latitude: $24^{\circ} 08^{\prime} 29^{\prime}$ ' S ; longitude: $49^{\circ} 49^{\prime} 45^{\prime \prime} \mathrm{W}$; e altitude $800 \mathrm{~m}$ ).

Foram utilizadas 13 árvores matriz selecionadas em uma população de Eucalyptus grandis plantados em um Latossolo Vermelho Escuro, no espaçamento de 3 × 2 m. Essas matrizes foram plantadas entre 1986 e 1987 e selecionadas entre 1992 e 1993, conforme apresentado no Quadro 1.

Quadro 1 - Caracterização das matrizes de Eucalyptus grandis selecionadas no Município de Arapoti, PR Table 1 - Characterization of Eucalyptus grandis donor trees selected in the Municipality of Arapoti, PR

\begin{tabular}{|c|c|c|c|c|c|c|c|}
\hline \multirow[t]{2}{*}{$\begin{array}{l}\text { Ano de } \\
\text { Plantio }\end{array}$} & \multirow[t]{2}{*}{$\begin{array}{l}\text { Data da } \\
\text { Seleção }\end{array}$} & \multirow[t]{2}{*}{$\begin{array}{c}\text { Área } \\
\text { Talhão (ha) }\end{array}$} & \multirow[t]{2}{*}{$\begin{array}{c}\text { № das Matrizes } \\
\text { Selecionadas }\end{array}$} & \multicolumn{2}{|c|}{$\begin{array}{c}\text { Média das Matrizes } \\
\text { Selecionadas } \\
\end{array}$} & \multicolumn{2}{|c|}{$\begin{array}{c}\text { Média do } \\
\text { Talhão } \\
\end{array}$} \\
\hline & & & & $\begin{array}{c}\text { dap } \\
(\mathrm{cm})\end{array}$ & $\begin{array}{l}H t \\
(\mathrm{~m}) \\
\end{array}$ & $\begin{array}{l}\text { dap } \\
(\mathrm{cm})\end{array}$ & $\begin{array}{c}H t \\
(\mathrm{~m})\end{array}$ \\
\hline 1986 & Nov./1992 & 29,8 & $\begin{array}{lllllllllll}27 & 28 & 30 & 31 & 32 & 37 & 38 & 42 & 44 & 46 & 47\end{array}$ & 26,3 & 32,2 & 15,5 & 26,0 \\
\hline 1987 & Jun./1993 & 28,8 & 8286 & 31,2 & 34,0 & 14,6 & 22,5 \\
\hline
\end{tabular}

R. Árvore, Viçosa-MG, v.30, n.5, p.737-747, 2006 
O desempenho silvicultural dos clones dessas matrizes foi avaliado por meio da instalação de um teste clonal no delineamento em blocos ao acaso, com três repetições e parcela quadrada de 25 plantas, em espaçamento de $3 \times 2 \mathrm{~m}$. As mudas utilizadas para instalação do teste clonal foram produzidas pela técnica de estaquia e em cuja implantação se utilizou uma fertilização inicial de NPK (5-30-10) na dosagem de 300 kg/há, na linha de plantio.

\subsection{Metodologia experimental}

\subsubsection{Características avaliadas - Árvores selecionadas}

Para a seleção das matrizes foram utilizadas as características dendrométricas: diâmetro à altura de 1,3 m (dap), altura total ( $H t)$, volume com casca $\left(\mathrm{V}_{\mathrm{CC}}\right)$, volume sem casca $\left(\mathrm{V}_{\mathrm{SC}}\right)$ e volume de casca $\left(\mathrm{V}_{\mathrm{C}}\right)$. Utilizouse também a avaliação das características fenotípicas: forma do fuste, espessura dos ramos, persistência dos ramos (desrama natural), tamanho da copa, tipo de casca e a presença de pragas e doenças.

Para verificar a superioridade (Sup.) da matriz selecionada em relação aos seus pares, em um raio de $10 \mathrm{~m}$ da árvore-alvo da seleção foram avaliadas as mesmas características dendrométricas em outras cinco árvores "co-dominantes" na área. Dessa forma, foram calculadas as superioridades em diâmetro à altura do peito $\left(\operatorname{Sup}_{d a p}\right)$, em altura $\left(\operatorname{Sup}_{H t}\right)$, em volume com casca $\left(\operatorname{Sup}_{\mathrm{V}_{c c}}\right)$, em volume sem casca $\left(\operatorname{Sup}_{\mathrm{vsc}_{\mathrm{sc}}}\right)$ e em volume de casca $\left(\operatorname{Sup}_{\mathrm{Vc}}\right)$, conforme apresentado a seguir:

$$
\text { Sup. }(\%)=100\left[\left(\mathrm{C}_{\mathrm{S}} / \mathrm{C}_{\mathrm{E}}\right)-1\right]
$$

$\mathrm{C}_{\mathrm{S}}=$ característica silvicultural da árvore selecionada; $\mathrm{e}$

$\mathrm{C}_{\mathrm{E}}=$ média das características das cinco árvores "codominantes".

O dap foi mensurado com o auxílio de uma fita diamétrica e a $\mathrm{Ht}$, obtida com o uso do relascópio. $\mathrm{O} \mathrm{V}_{\mathrm{CC}}$ e o $\mathrm{V}_{\mathrm{SC}}$ foram calculados através da equação de múltiplos volumes de Leite et al. (1995), utilizada pela empresa para mensuração de seus povoamentos de Eucalyptus grandis, conforme apresentado a seguir:

$\mathrm{V}=0,000048 d a p^{1,720483} H t^{1,180736} \mathrm{e}^{(-3,00555)(\mathrm{x} / \mathrm{dap})}\left\{1-(\mathrm{d} / d a p)^{1+0,228531 \mathrm{~d}}\right\}$ em que:

$d a p=$ diâmetro a 1,3 $\mathrm{m}$ de altura;

$H t=$ altura total; $\mathrm{tx}=0$, para volume com casca e 1 para volume sem casca;

$\mathrm{d}=$ diâmetro comercial superior.

$\mathrm{O}$ volume de casca $\left(\mathrm{V}_{\mathrm{C}}\right)$ foi calculado pela diferença entre o $\mathrm{V}_{\mathrm{CC}}$ e o $\mathrm{V}_{\mathrm{SC}}$

\subsubsection{Análise multivariada - Árvores selecionadas}

As matrizes selecionadas foram agrupadas pelo método de Tocher, tendo como base a distância euclidiana média, conforme descrito em Cruz e Regazzi (1997) e Valentin (2000).

Para a confirmação do agrupamento e obtenção das informações em nível de indivíduos, realizou-se a dispersão dos escores das variáveis em eixos cartesianos, estabelecidos por componentes principais, conforme metodologia descrita em Valentin (2000).

Para verificar a importância relativa das características utilizadas para a formação dos grupos, foi utilizado o método proposto por Singh (1981), citado por Cruz e Carneiro (2003).

Para estimar a probabilidade de árvores classificadas corretamente dentro dos diferentes grupos formados pelo método de Tocher e para estabelecer equações de discriminante que permitam classificar árvores-matriz selecionadas posteriormente dentro dos grupos prédefinidos pelo método de Tocher, foi empregada a análise de discriminante proposta por Anderson (1958). Segundo Cruz e Carneiro (2003), considerando, por exemplo, apenas duas populações, ter-se-ia:

$\pi_{1} e \pi_{2}$ populações 1 e 2 , respectivamente; $\mu_{1} e$ $\mu_{2}$ vetor de médias dos p caracteres avaliados em $\pi_{1}$ $e \pi_{2}$, respectivamente.

$\omega_{1} e \omega_{2}$ matriz de co-variâncias entre os caracteres avaliados em $\pi_{1} e \pi_{2}$, respectivamente.

$\rho_{1}$ e $\rho_{2}$ probabilidades, "a priori”, de os indivíduos pertencerem a $\pi_{1}$ e $\pi_{2}$, respectivamente.

$X=$ vetor de variáveis representativas dos caracteres envolvidos na análise.

Classifica-se o i-ésimo material genético com vetor de média $X_{i}$ na população $\pi_{j}$ se, e somente se, $D_{j}\left(\mathrm{x}_{i}\right)$ for o maior entre os elementos do conjunto $\left\{D_{1}\left(\mathrm{x}_{i}\right)\right.$, $D_{2}\left(\mathrm{x}_{i}\right)$. Utilizando-se as funções discriminantes e os dados das próprias populações, $\pi_{1} e \pi_{2}$ ainda é possível estimar a taxa de erro aparente que mede a eficiência da função discriminante em classificar os genótipos, corretamente, nas populações previamente estabelecidas.

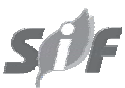

R. Árvore, Viçosa-MG, v.30, n.5, p.737-747, 2006 


\subsubsection{Avaliações no teste clonal}

Aos 7 anos de idade do teste clonal foram mensurados o dap e a $H t$ das nove plantas centrais da parcela, com o auxílio de uma fita diamétrica e do relascópio, respectivamente. $\mathrm{O} \mathrm{V}_{\mathrm{CC}}$ e o $\mathrm{V}_{\mathrm{SC}}$ foram calculados através da equação de múltiplos volumes de Leite et al. (1995), conforme descrito no item 2.2.1.

O incremento médio anual (IMA) foi calculado de acordo com o estande de plantas por hectare e a idade de avaliação do teste clonal.

Os dados obtidos foram submetidos à análise de variância e as médias, discriminadas pelo teste de Scott e Knott.

\subsubsection{Desempenho da árvore selecionada $x$ desempenho do clone}

Para verificar o efeito das características utilizadas para formação dos grupos sobre a produtividade em incremento médio anual (IMA) das matrizes selecionadas após a avaliação do teste clonal, estas foram dispostas em um "ranking" de classificação, sendo também realizada a análise de trilha, conforme descrito em Cruz e Regazzi (1997).

\section{RESULTADOS E DISCUSSÕES}

\subsection{Análise multivariada - Árvores selecionadas}

A análise de agrupamento das 13 árvores selecionadas foi realizada com base no dap, $\operatorname{Sup}_{d a p}$, $H t$ e na Sup ${ }_{H t}$, uma vez que o volume apresentou forte correlação com essas características para a aplicação da análise de agrupamento (GARCIA, 1998). Após o agrupamento, formaram-se três grupos distintos, indicando a existência de variabilidade entre as matrizes selecionadas (Quadro 2).

Quadro 2-Agrupamento das 13 árvores-matriz de Eucalyptus grandis, tendo como base as características dap, $H t$ Sup $_{d a p}$ e $\operatorname{Sup}_{H t}$ e utilizando a distância euclidiana média e o método de otimização de Tocher

Table 2-Clustering of 13 Eucalyptus grandis donor trees as a function of dap, Ht, Sup dap and Sup ${ }_{H t}$ using the Euclidean distance and the Tocher's optimization procedure

\begin{tabular}{|c|c|c|}
\hline Grupo & № de Matrizes & № das Matrizes \\
\hline I & 8 & $\begin{array}{llllllll}28 & 30 & 31 & 32 & 37 & 38 & 42 & 44\end{array}$ \\
\hline II & 3 & 278286 \\
\hline III & 2 & 4647 \\
\hline
\end{tabular}

dap: diâmetro à altura do peito, $H t$ : altura comercial, $\operatorname{Sup}_{d a p}$, : superioridade em dap e $\operatorname{Sup}_{H t}$, superioridade em altura.
Num programa de silvicultura clonal, a seleção de clones dentro dos diferentes grupos tende a aumentar a variabilidade genética na área de plantio, diminuindo a suscetibilidade dos plantios clonais, aos possíveis riscos associados a eles, como o estreitamento excessivo da base genética, tornando-os pouco flexíveis às mudanças na demanda do mercado consumidor e às variações ambientais (ASSIS, 1996; XAVIER, 2003); e a vulnerabilidade ao ataque de pragas e doenças.

A seleção de indivíduos dentro de um mesmo grupo pode, no entanto, ser útil, caso seja possível predizer o comportamento desses grupos no futuro, após as avaliações de sua "performance" em testes clonais e plantios comerciais, tanto em critérios de produtividade quanto de qualidade da madeira.

Lopes (1992) e Ferreira (1994) relataram a falta de estudos básicos sobre o agrupamento de clones em função de suas afinidades, ressaltam que resultados obtidos através da análise de agrupamento podem propiciar o ordenamento de materiais genéticos possível de serem utilizado em testes clonais. Isso facilitaria o entendimento das inter-relações entre os clones, as condições ambientais e as características tecnológicas da madeira (DEMUNER e BERTOLUCCI, 1994; GARCIA, 1998).

Em relação à análise de componentes principais, verificou-se que os dois primeiros componentes explicaram $86,89 \%$ da variância total das características avaliadas (Quadro 3). Segundo Cruz e Regazzi (1997), o estudo da diversidade genética por meio da dispersão gráfica dos escores gerados pelos componentes principais, em espaço bidimensional, é aceitável quando a variância dos dois primeiros componentes explica acima de $80 \%$ da variância total. Dessa forma, optouse por apresentar a dispersão dos escores gerados num gráfico com os dois componentes em questão.

Segundo critérios adotados por Cruz e Regazzi (1997) e Pereira et al. (2003), o dap e a $\operatorname{Sup}_{H t}$, por apresentarem o maior coeficiente nos últimos componentes principais, que explicam pequena parcela da variância total, poderiam ser descartados. Porém,

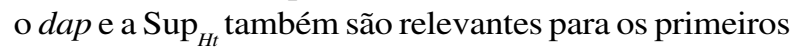
componentes principais, o que, segundo comentários de Morais (1992), torna questionável o descarte dessas variáveis. Dessa forma, optou-se por não proceder ao descarte de variáveis para o restante da análise. 
Quadro 3 - Estimativas das variâncias (autovalores) associadas aos componentes principais e os respectivos coeficientes de ponderação (autovetores) das características avaliadas na seleção das árvores-matriz de Eucalyptus grandis, na região de Arapoti, PR

Table 3 - Estimates of variances (eigenvalues) associated with principal components and respective ponderation coefficients (eigenvectors) of characteristics evaluated for Eucalyptus grandis donor tree selection, in Arapoti, PR

\begin{tabular}{lccccrr}
\hline $\begin{array}{l}\text { Componente } \\
\text { Principal }\end{array}$ & $\begin{array}{c}\text { Variância } \\
\text { (autovalor) }\end{array}$ & $\begin{array}{c}\text { Variância } \\
\text { Acumulada (\%) }\end{array}$ & \multicolumn{3}{c}{$\begin{array}{c}\text { Coeficientes de Ponderação } \\
\text { Associados a: }\end{array}$} \\
\cline { 4 - 7 } & & & $d a p$ & Sup $_{\text {dap }}$ & $H_{t}$ & Sup $_{H t}$ \\
\hline CP1 & 2,7863 & 69,65 & 0,5390 & 0,4500 & 0,5081 & 0,4988 \\
CP2 & 0,6890 & 86,89 & 0,2143 & 0,7431 & $-0,4859$ & $-0,4071$ \\
CP3 & 0,3509 & 95,66 & $-0,4966$ & 0,2661 & $-0,4120$ & 0,7162 \\
CP4 & 0,1735 & 100,00 & 0,6458 & $-0,4176$ & $-0,5796$ & 0,2695 \\
\hline
\end{tabular}

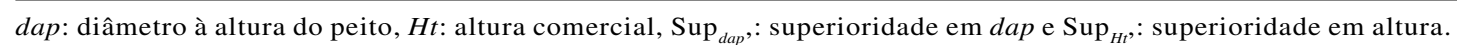

A dispersão gráfica dos escores dos dois primeiros componentes principais indicou boa relação entre os grupos formados pelo método de Tocher (Quadro 2) e a dispersão dos escores no plano bidimensional (Figura 1).

Quanto à importância relativa das características avaliadas para a separação dos grupos, observou-se que a $\operatorname{Sup}_{d a p}$ foi a que mais contribuiu (Figura 2), evidenciando a importância da obtenção de uma estimativa da superioridade das matrizes selecionadas em relação aos seus pares, para a sua separação em grupos divergentes.

No que tange às árvores-matriz mais divergentes pela análise visual, ressaltam-se as distâncias entre as matrizes 37 (grupo 1), 46 (grupo III) e 82 (grupo

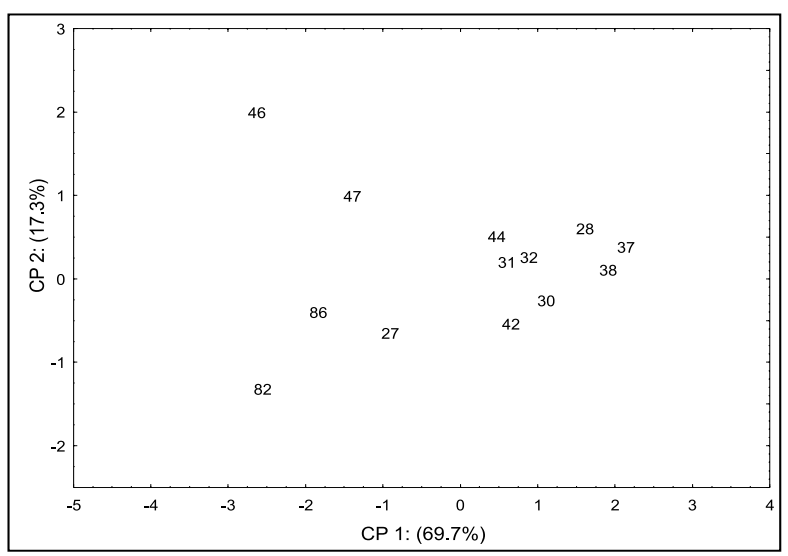

Figura 1 - Dispersão de escores das 13 árvores-matriz de Eucalyptus grandis, selecionadas na região de Arapoti, PR, em relação aos dois primeiros componentes principais (CP1 e CP2).

Figure 1 -Score dispersion of Eucalyptus grandis donor trees, selected in Arapoti - PR, in relation to the two first principal components $(C P 1)$ and $(C P 2)$
II). Assim, se o objetivo for aumentar a variabilidade genética na área de plantio, visando reduzir os possíveis riscos de estreitamento da base genética oriundos da clonagem, essas matrizes devem ser consideradas. Destaca-se que esses materiais genéticos devem, ainda, ser avaliados em teste clonal e apresentar bom desempenho silvicultural e de qualidade da madeira, para serem utilizados comercialmente.

NaFigura3, observam-se os dados das variáveis utilizadas para a separação dos grupos. No que tange ao dap, o grupo que apresentou a maior média foi o III $(30,2 \mathrm{~cm})$.

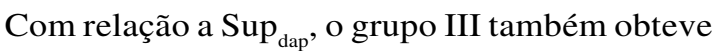
a melhor "performance", com 41,6\%, indicando que, além de ser o melhor grupo em dap, a dominância das árvores desse grupo em relação aos seus pares também foi a mais expressiva. Quanto à característica $H t$, o grupo II que apresentou a maior média $(35,1 \mathrm{~m})$ também se destacou em $\operatorname{Sup}_{H t}(12,8 \%)$.

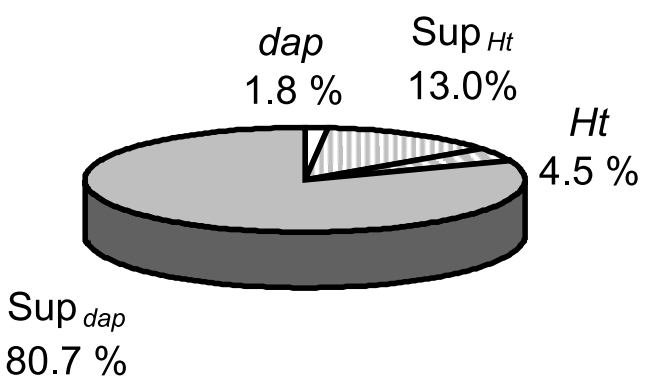

Figura 2-Importância relativa das características para separação dos grupos, utilizando-se como medida de dissimilaridade a distância euclidiana média e o método de agrupamento de Tocher.

Figure 2-Relative importance of characteristics for group separation using Euclidean distance and the Tocher's method as dissimilarity measure

R. Árvore, Viçosa-MG, v.30, n.5, p.737-747, 2006 

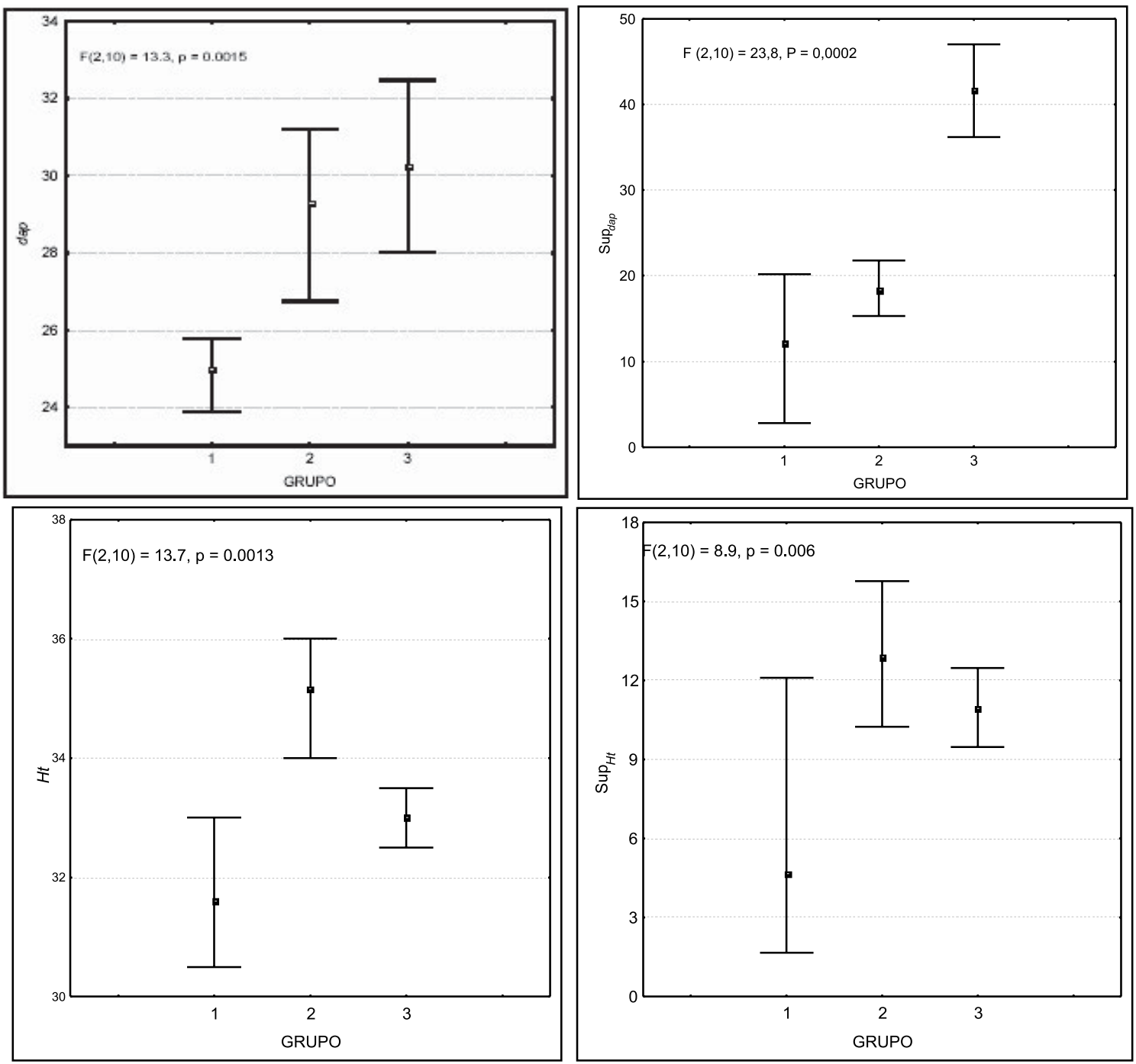

Figura 3 - Média, mínimo e máximo das características (dap, $\operatorname{Sup}_{d a p}, H t, \operatorname{Sup}_{H}$ ) dos três grupos formados pelo método de Tocher. Figure 3 - Average, minimum and maximum values of dap, $\mathrm{Sup}_{d a p}$, $\mathrm{Ht}$, Sup ${ }_{H t}$ of the three groups formed by the Tocher's method.

A análise de correlação de Pearson (Quadro 4) confirma esses resultados, pois demonstra que árvores com maiores valores para dap e $H t$ tendem a apresentar maior superioridade para essas características em relação às árvores vizinhas. Provavelmente, isso ocorreu por efeitos oriundos da competição entre os diferentes genótipos pelos recursos presentes na área.

\subsubsection{Análise de discriminante}

Com base na análise discriminante, observou-se que todos os indivíduos foram classificados dentro dos respectivos grupos (Quadro 5), indicando a precisão da técnica de agrupamento utilizada, no caso o método de Tocher. Com isso, as funções discriminantes obtidas (Quadro 6) podem ser utilizadas para classificar novas árvores entre os grupos em futuras seleções de árvoresmatriz (LOPES, 2003).

Segundo Malhotra (2001), o modelo de análise discriminante de Anderson (1958) envolve combinações lineares da forma: $D=\beta_{0}+\beta_{1} X_{1}+\beta_{2} X_{2}+\beta_{3} X_{3}+$ $\ldots+\beta_{k} X_{k}$, em que $\mathrm{D}$ é igual ao escore discriminante, $\beta$ é o coeficiente ou peso discriminante e $X$ representa a i-ésima variável preditora ou independente. Assim, para classificar um novo indivíduo dentro de um dos 
grupos, deverão ser mensuradas as quatros variáveis (dap, Sup dap,$H t$ e $\operatorname{Sup}_{H t}$ ), substituindo-se os valores na função discriminante referente a cada grupo, e o grupo que obtiver o maior valor D será aquele para que a nova árvore deverá ser alocada.

Dessa forma, com a possibilidade de alocar novas árvores dentro dos diferentes grupos formados pelo método de Tocher, em futuras seleções de matrizes para clonagem será possível ter um indicativo das afinidades genéticas entre eas. Com base nessas informações, pode-se orientar a distribuição espacial das árvores mais divergentes em pomares de sementes clonais, visando aumentar o efeito da heterose, oriunda do cruzamento entre materiais geneticamente divergentes e com bom desempenho silvicultural.

\subsection{Avaliações do teste clonal}

Com base no Quadro 7, observa-se um comportamento diferenciado entre os clones com relação a todas as características avaliadas, evidenciando diferenças significativas entre os diferentes materiais genéticos utilizados no teste clonal.

Quadro 4 - Correlação de Pearson entre as características avaliadas para a seleção da árvore-matriz

Table 4- Pearson's correlation between the studied characteristics for donor tree selection

\begin{tabular}{cccc}
\hline Características & $\operatorname{Sup}_{d a p}$ & $H t$ & $\operatorname{Sup}_{H t}$ \\
\hline dap & $0,69^{*}$ & $0,69^{*}$ & $0,59^{*}$ \\
$\operatorname{Sup}_{d a p}$ & & $0,39^{\mathrm{ns}}$ & $0,46^{\mathrm{ns}}$ \\
$H t$ & & & $0,62^{*}$ \\
\hline
\end{tabular}

* e ${ }^{\text {ns }}$ Significativos e não-significativos pelo teste de t a $5 \%$ de probabilidade.
No Quadro 8, observa-se que os clones 28, 47 e 82 se destacaram em relação aos demais quanto às características avaliadas. Esses três clones, além de apresentarem o melhor desempenho silvicultural per si, pertencem a grupos diferentes (Quadro 2), apresentando, portanto, divergência genética. Devido a essa divergência, aliada à alta produtividade, esses clones seriam ainda os prioritários para a alocação dentro de um pomar de semente, visando aproveitar a heterose manifestada no cruzamento de genitores divergentes.

Quadro 5 - Número de casos e porcentagens de classificação das árvores nos três grupos obtidos pelo método de Tocher

Table 5 - Number of cases and percent classification of trees in the three groups obtained by the Tocher's method

\begin{tabular}{ccccc}
\hline Grupo & No de Árvores/(\%) & \multicolumn{3}{c}{ Grupo } \\
\cline { 3 - 5 } & & I & II & III \\
\hline I & 8 & 8 & \\
& $100 \%$ & $100 \%$ & 3 & \\
II & 3 & & $100 \%$ & 2 \\
& $100 \%$ & & $100 \%$ \\
III & 2 & & \\
& $100 \%$ & & & \\
& Taxa de erro aparente $=\mathbf{0 \%}$ \\
\hline
\end{tabular}

Quadro 6 - Equações de discriminante dos grupos formados pelo método de Tocher

Table 6-Discriminant functions of groups formed by the Tocher's method

\begin{tabular}{cc}
\hline Grupo & Equações $^{\text {I }}$ \\
\hline II & $\mathrm{D}=-0,538$ dap $-0,107 \mathrm{Sup}_{d a p}+20,1 H t-4,8 \mathrm{Sup}_{H t}-301,2$ \\
III & $\mathrm{D}=-0,236$ dap $-0,181 \mathrm{Sup}_{d a p}+20,8 \mathrm{Ht}-4,6 \mathrm{Sup}_{H t}-332,4$ \\
\hline
\end{tabular}

Quadro 7 - Análise de variância das características diâmetro à altura do peito (dap), altura total (Ht), volume com casca $\left(\mathrm{V}_{\mathrm{CC}}\right)$, volume sem casca $\left(\mathrm{V}_{\mathrm{SC}}\right)$, volume de casca $\left(\mathrm{V}_{\mathrm{C}}\right)$ e incremento médio anual em $\mathrm{m}^{3} / \mathrm{ha} / \mathrm{ano}$ sem casca (IMA) dos clones de Eucalyptus grandis, avaliados em teste clonal aos 7 anos de idade

Table 7 - Analysis of variance for the characteristics diameter (dap), total height (Ht), volume with bark, ( $\left.V_{C C}\right)$, volume without bark $\left(V_{S C}\right)$ and bark volume $\left(V_{C}\right)$ and average annual increase in $m^{3} /$ ha/year without bark (IMA) of Eucalyptus grandis clones evaluated at 7 year of age

\begin{tabular}{|c|c|c|c|c|c|c|c|}
\hline \multirow[t]{2}{*}{ FV } & \multirow[t]{2}{*}{ GL } & \multicolumn{5}{|c|}{ Quadrado Médio } & \multirow[b]{2}{*}{ IMA $\left(\mathrm{m}^{3} / \mathrm{ha} /\right.$ ano $)$} \\
\hline & & $\operatorname{dap}(\mathrm{cm})$ & $H t(\mathrm{~m})$ & $\mathrm{V}_{\mathrm{CC}}\left(\mathrm{m}^{3}\right)$ & $\mathrm{V}_{\mathrm{SC}}\left(\mathrm{m}^{3}\right)$ & $V_{C}\left(m^{3}\right)$ & \\
\hline Bloco & 2 & 6,2 & 11,6 & 0,011 & 0,009 & 0,0001 & 538,6 \\
\hline Clone & 12 & $9,5 *$ & $18,9 *$ & $0,031 *$ & $0,025 *$ & $0,0002 *$ & $1.469,6 *$ \\
\hline Resíduo & 24 & 2,4 & 7,3 & 0,007 & 0,005 & 0,007 & 337,6 \\
\hline Média geral & - & 18,2 & 29,8 & 0,4481 & 0,3863 & 0,0611 & 91,9 \\
\hline $\mathrm{CV}_{\mathrm{exp}} \cdot(\%)$ & - & 8,5 & 9,0 & 19,0 & 19,9 & 14,3 & 19,9 \\
\hline
\end{tabular}

* Significativo pelo teste $\mathrm{F}$ a $5 \%$ de probabilidade. 
Quadro 8 - Média das características diâmetro à altura do peito (dap), altura total $(H t)$, volume com casca $\left(\mathrm{V}_{\mathrm{CC}}\right)$, volume sem casca $\left(\mathrm{V}_{\mathrm{sC}}\right)$, volume de casca e incremento médio anual de madeira sem casca (IMA) doaclones de Eucalyptus grandis, avaliados aos 7 anos de idade

Table 8 - Means of the characteristics diameter (dap), total height (Ht), volume with bark, $\left(V_{C C}\right)$, volume without bark $\left(V_{S C}\right)$ and bark volume $\left(V_{C}\right)$ and average annual increase of wood without bark (IMA) of Eucalyptus grandis clones evaluated at 7 year of age

\begin{tabular}{|c|c|c|c|c|c|c|}
\hline Clone & dap $(\mathrm{cm})$ & $H t(\mathrm{~m})$ & $\mathrm{V}_{\mathrm{CC}}\left(\mathrm{m}^{3}\right)$ & $\mathrm{V}_{\mathrm{SC}}\left(\mathrm{m}^{3}\right)$ & $\mathrm{V}_{\mathrm{C}}\left(\mathrm{m}^{3}\right)$ & IMA (m³/ha/ano) \\
\hline 27 & $18,4 \mathrm{~b}$ & $30,7 \mathrm{a}$ & $0,4445 \mathrm{~b}$ & $0,3812 \mathrm{~b}$ & $0,0633 \mathrm{~b}$ & $90,7 \mathrm{~b}$ \\
\hline 28 & $21,8 \mathrm{a}$ & 32,8 a & $0,6382 \mathrm{a}$ & $0,5603 \mathrm{a}$ & 0,0779 a & 133,3 a \\
\hline 30 & $17,4 \mathrm{~b}$ & $31,7 \mathrm{a}$ & $0,4140 \mathrm{~b}$ & $0,3513 \mathrm{~b}$ & $0,0626 \mathrm{~b}$ & 83,6 b \\
\hline 31 & $16,8 \mathrm{~b}$ & 28,0 a & $0,3902 \mathrm{~b}$ & $0,3348 \mathrm{~b}$ & $0,0555 \mathrm{~b}$ & $79,7 \mathrm{~b}$ \\
\hline 32 & $16,0 \mathrm{~b}$ & 25,6 a & $0,3368 \mathrm{~b}$ & $0,2881 \mathrm{~b}$ & $0,0487 \mathrm{~b}$ & $68,6 \mathrm{~b}$ \\
\hline 37 & $16,6 \mathrm{~b}$ & 25,3 a & $0,3208 \mathrm{~b}$ & $0,2728 \mathrm{~b}$ & $0,0480 \mathrm{~b}$ & $64,9 \mathrm{~b}$ \\
\hline 38 & $16,0 \mathrm{~b}$ & 29,1 a & $0,3146 \mathrm{~b}$ & $0,2622 \mathrm{~b}$ & $0,0524 \mathrm{~b}$ & $62,4 \mathrm{~b}$ \\
\hline 42 & $18,1 \mathrm{~b}$ & 29,8 a & $0,4701 b$ & $0,4071 \mathrm{~b}$ & $0,0631 \mathrm{~b}$ & $96,9 \mathrm{~b}$ \\
\hline 44 & 18,9 a & 29,0 a & $0,4641 \mathrm{~b}$ & $0,4028 \mathrm{~b}$ & $0,0613 \mathrm{~b}$ & $95,9 \mathrm{~b}$ \\
\hline 46 & $18,6 \mathrm{~b}$ & $31,5 \mathrm{a}$ & $0,4617 \mathrm{~b}$ & $0,3964 \mathrm{~b}$ & $0,0653 \mathrm{~b}$ & $94,3 \mathrm{~b}$ \\
\hline 47 & 19,8 a & 33,9 a & $0,5664 \mathrm{a}$ & $0,4914 \mathrm{a}$ & $0,0750 \mathrm{a}$ & 116,9 a \\
\hline 82 & 20,6 a & 30,8 a & $0,5990 \mathrm{a}$ & $0,5267 \mathrm{a}$ & $0,0723 \mathrm{a}$ & 125,3 a \\
\hline 86 & $16,8 \mathrm{~b}$ & $29,2 \mathrm{a}$ & $0,4302 \mathrm{~b}$ & $0,3707 \mathrm{~b}$ & $0,0594 \mathrm{~b}$ & 88,2 b \\
\hline
\end{tabular}

Médias seguidas pela mesma letra na coluna constituem um grupo homogêneo segundo o critério de Scott e Knott a 5\% de probabilidade.

\subsubsection{Desempenho da árvore selecionada $x$ desempenho do clone}

De acordo com a posição dos materiais genéticos no "ranking" de classificação (Quadro 9), observase que, com exceção das matrizes 28 e 46, existe certa concordância entre as características avaliadas na seleção inicial das árvores-matriz (dap, $\operatorname{Sup}_{d a p}, H t, \operatorname{Sup}_{H t}$ ) e o seu desempenho no teste clonal em incremento médio anual (IMA). Esse fato também foi comprovado pela análise de trilha.

Já o fato de não se verificar esse comportamento em todos os materiais genéticos e não haver relação maior entre as características pode estar relacionado a fatores da interação clone x ambiente. A saber: padrão de qualidade de mudas, locais diferentes de seleção da árvore-matriz e plantio do clone, variações climáticas ao longo da rotação da cultura, efeitos oriundos da competição intraclonal e variações no manejo silvicultural empregado pela empresa. Tal fato torna indispensável a avaliação dos materiais genéticos em testes clonais, para que efetivamente possam ser selecionados os melhores clones.

A análise de trilha evidenciou relação significativa entre as características avaliadas para a seleção da árvore-matriz e o seu desempenho no teste clonal (Quadro 10). Nota-se que a variável $\operatorname{Sup}_{H t}$ apresentou predomínio sobre as demais para o estabelecimento da correlação, indicando que, dentro da amplitude dos valores mensurados na seleção inicial das árvores-matriz (Figura 3), o fato de selecionar uma árvore com maior valor para essa característica é um bom indicativo do seu bom desempenho após a sua avaliação em teste clonal.

Quadro 9-Ranking das características utilizadas para a seleção das 13 árvores-matri (dap, Ht, $\left.\operatorname{Sup}_{d a p}, \operatorname{Sup}_{H t}\right) \mathrm{e}$ o desempenho dessas matrizes no teste clonal em incremento médio anual (IMA)

Table 9 - Ranking of characteristics used for selection of 13 donor trees (dap, $\mathrm{Ht}$, Sup ${ }_{\text {dap }}$, Sup $_{\mathrm{Ht}}$ ) and their performances at the clonal test, in average annual increase (IMA)

\begin{tabular}{|c|c|c|c|c|c|}
\hline \multirow[t]{3}{*}{ Clone } & \multicolumn{5}{|c|}{ Posição no RanKing } \\
\hline & \multirow[b]{2}{*}{ dap } & \multicolumn{3}{|c|}{ Árvore Selecionada } & \multirow{2}{*}{$\frac{\text { Clone }}{\text { IMA }^{1}}$} \\
\hline & & $\operatorname{Sup}_{d a p}$ & $H t$ & $\operatorname{Sup}_{H t}$ & \\
\hline 27 & $5^{\circ}$ & $5^{\circ}$ & $3^{\underline{0}}$ & $2^{\underline{o}}$ & 7 음 \\
\hline 28 & 60 & $11^{\mathrm{o}}$ & $11^{\circ}$ & $11^{\mathrm{o}}$ & $1 \stackrel{\mathrm{o}}{1}$ \\
\hline 30 & $12^{\circ}$ & $10^{\circ}$ & $5 \stackrel{\circ}{2}$ & $9 \circ$ & 9 은 \\
\hline 31 & $10^{\circ}$ & $6^{\mathrm{o}}$ & $6^{\circ}$ & $8^{0}$ & $10^{\underline{o}}$ \\
\hline 32 & 9은 & $8^{\circ}$ & $7 \stackrel{0}{0}$ & $10^{\mathrm{o}}$ & $11^{\mathrm{o}}$ \\
\hline 37 & $11^{\mathrm{o}}$ & $12^{\mathrm{o}}$ & $12^{\mathrm{o}}$ & $13^{\mathrm{o}}$ & $12^{\mathrm{o}}$ \\
\hline 38 & $7 \underline{0}$ & $13^{\circ}$ & $13^{\circ}$ o & $12^{\circ} \mathrm{o}$ & 13 음 \\
\hline 42 & 13 은 & 9은 & $10^{\circ}$ & $4^{\circ}$ & $4 \stackrel{\circ}{2}$ \\
\hline 44 & $8^{\circ}$ & $4 \stackrel{\circ}{2}$ & $9^{\circ}$ & 7 o & $5^{\circ}$ \\
\hline 46 & $1 \stackrel{\circ}{\circ}$ & $1 \stackrel{\mathrm{o}}{1}$ & 4 o & $6^{\circ}$ & 60 \\
\hline 47 & $4 \stackrel{\circ}{\circ}$ & $2^{\mathrm{o}}$ & 8 은 & 3 o & 3 o \\
\hline 82 & $2^{\circ}$ & $7 \stackrel{0}{ }$ & $1^{\circ} \mathrm{o}$ & $1^{\circ} \stackrel{\circ}{ }$ & $2^{\circ}$ \\
\hline 86 & 3음 & 3음 & $2 \underline{\circ}$ & 5음 & 80 \\
\hline
\end{tabular}

dap: diâmetro à altura do peito, $H t$ : altura comercial, $\operatorname{Sup}_{d a p}$,: superioridade em dap e $\operatorname{Sup}_{H t}$, superioridade em altura.

${ }^{1}$ Incremento médio anual em metro cúbico de madeira sem casca. 
Quadro 10 - Estimativas dos efeitos diretos e indiretos das características utilizadas para seleção inicial da árvore-matriz (dap, $\operatorname{Sup}_{d a p}, H t, \operatorname{Sup}_{H t}$ ) e o desempenho dessas matrizes no teste clonal em incremento médio anual em volume sem casca (IMA), obtidas a partir dos dados transformados para a escala logarítmica

Table 10-Estimates of direct and indirect effects of characteristics used for initial selection of donor trees (dap, $\mathrm{Ht}$, Sup $_{d a p}$, Sup $_{H t}$ ) and their performances at the clonal test, in average annual increase of volume without bark (IMA), obtained from data transformed to logarithmic scale

\begin{tabular}{|c|c|c|c|}
\hline \multirow{2}{*}{ dap } & \multicolumn{2}{|l|}{ Caracteres } & Estimativa \\
\hline & & & \\
\hline & Efeito direto sobre IMA & 0.13 & \\
\hline & Efeito indireto via $\operatorname{Sup}_{d a p}$ & 0.09 & \\
\hline & Efeito indireto via $H t$ & -0.09 & \\
\hline & Efeito indireto via $\operatorname{Sup}_{H t}$ & 0.45 & \\
\hline & Total & & 0.58 \\
\hline \multicolumn{4}{|c|}{$\operatorname{Sup}_{d a p}$} \\
\hline & Efeito direto sobre IMA & 0.24 & \\
\hline & Efeito indireto via dap & 0.05 & \\
\hline & Efeito indireto via $H t$ & -0.06 & \\
\hline & Efeito indireto via $\operatorname{Sup}_{H t}$ & 0.47 & \\
\hline & Total & & 0.70 \\
\hline \multirow[t]{5}{*}{$H t$} & Efeito direto sobre IMA & -0.12 & \\
\hline & Efeito indireto via dap & 0.10 & \\
\hline & Efeito indireto via $\operatorname{Sup}_{d a p}$ & 0.13 & \\
\hline & Efeito indireto via $\operatorname{Sup}_{H t}$ & 0.50 & \\
\hline & Total & & 0.61 \\
\hline \multicolumn{4}{|l|}{$\operatorname{Sup}_{H t}$} \\
\hline & Efeito direto sobre IMA & 0.73 & \\
\hline & Efeito indireto via dap & 0.08 & \\
\hline & Efeito indireto via $\operatorname{Sup}_{d a p}$ & 0.15 & \\
\hline & Efeito indireto via $\mathrm{Ht}$ & -0.08 & \\
\hline & Total & & 0.88 \\
\hline & iente de dete & & \\
\hline Efeit & residual & & \\
\hline
\end{tabular}

dap: diâmetro à altura do peito; $H t$, altura comercial; $\operatorname{Sup}_{d a p}$, superioridade em dap Sup $_{H t}$, : superioridade em altura.

Além de apresentar a maior correlação total, a Sup $\operatorname{Su}_{H t}$ exibiu o maior efeito direto sobre o incremento médio anual (IMA), superando as demais características avaliadas. Com relação aos efeitos indiretos, a $\operatorname{Sup}_{H t}$ também apresentou os maiores valores e positivos, o que implica uma correlação de caracteres em sentido favorável. Dessa forma, a seleção simultânea via indireta, realizada com base nessa característica, aponta para uma alta eficiência no processo seletivo (KUREK et al., 2001).

Em futuras seleções de árvores-matriz para clonagem, pode-se, assim, dar preferência a seleção de árvores que sejam alocadas para grupos que apresentem melhor desempenho para a característica $\operatorname{Sup}_{H t}$, o que neste trabalho pode ser feito pelas equações de discriminante do Quadro 6.

Dessa forma, mesmo com a necessidade de avaliação das matrizes selecionadas em teste clonal a aplicação da metodologia proposta poderá diminuir substancialmente o número de clones a serem avaliados em teste, racionalizando o uso da clonagem nas empresas florestais. Uma vez que a etapa de avaliação das matrizes selecionadas para clonagem tende a apresentar-se como a mais onerosa e demorada para a silvicultura clonal, devido ao tamanho das parcelas a serem empregadas, ao alto custo de implantação, à baixa disponibilidade de mudas, às limitações de área, ao aumento da área a ser controlada e às avaliações periódicas, entre outros (PEREIRA, 1996; ANDRADE et al., 1997; SILVA, 2001).

\section{CONCLUSÕES}

Com base nos resultados obtidos neste trabalho, pode-se concluir que principalmente, a avaliação da superioridade em $H t\left(\operatorname{Sup}_{H t}\right)$ da árvore-matriz em relação às árvores "co-dominantes" do local de seleção é um procedimento importante para a escolha inicial dessas árvores. No entanto, a instalação de testes clonais é imprescindível para a silvicultura clonal, principalmente devido à existência da interação do genótipo com os efeitos ambientais.

A análise multivariada é eficaz para a alocação das matrizes em grupos divergentes, bem como para a classificação de árvores-matriz selecionadas posteriormente dentro dos grupos pré-definidos pelo método de Tocher.

\section{AGRADECIMENTOS}

À CAPES, pela concessão da bolsa de estudos durante o Mestrado; à International Paper, pela cessão dos dados experimentais para a dissertação; e à KLABIN S.A., pelo apoio para a conclusão deste artigo.

\section{REFERÊNCIAS BIBLIOGRÁFICAS}

Anderson, T. W. An introduction to multivariate statistical analysis. New York: John Wiley, 1958. 374 p.

R. Árvore, Viçosa-MG, v.30, n.5, p.737-747, 2006 
ANDRADE, H. B. et al. Avaliação da eficiência de utilização de bordaduras internas em teste clonais. In. IUFRO CONFERENCE ON SILVICULTURE AND IMPROVEMENT OF EUCALYPTS, 1997, Salvador-BA. Anais... Colombo: EMBRAPA/ CNPFlorestas, 1997. v. 1. p.91-94.

ANDRADE, H. B. Eficiência dos experimentos com clones na cultura do eucalipto. 2002. 162 f. Tese (Doutorado em Agronomia) - Universidade Federal de Lavras, Lavras, 2002.

ASSIS, T. F. Melhoramento genético do Eucalipto. Informe Agropecuário, v. 18, n. 185. p. 32-21, 1996.

BARREIROS, R. M. et al. Modelo de otimização para seleção de árvores matrizes de Eucalyptus grandis. Scientia Forestalis, n. 61, p. 25-39, 2002.

CAMPINHOS Jr., E.; IKEMORI, J. K. Clonagem de Eucalyptus spp. na Aracruz Florestal S/A. Problemática da produção de mudas em essências florestais. IPEF, v. 4, n. 13, p. 6-11, 1987. (Série Técnica).

CRUZ, C. D.; REGAZZI, A. J. Modelos

biométricos aplicados ao melhoramento genético. Viçosa, MG: Universidade Federal de Viçosa, 1997. 390 p.

CRUZ, C. D.; CARNEIRO, P. C. S. Modelos biométricos aplicados ao melhoramento genético. Viçosa, MG: Universidade Federal de Viçosa, 2003. 585 p.

DEMUNER, B. J.; BERTOLUCCI, F. L. G. Seleção florestal: uma nova abordagem a partir de parâmetros genéticos e fenotípicos para características da madeira e polpa do eucalipto. O Papel, v. 55, n. 1, p. 16-23, 1994.

FERREIRA, M. Melhoramento e a silvicultura intensiva clonal. IPEF, v. 45, p. 22-30, 1992.

FERREIRA, M. Características da madeira de espécies/procedências/árvores superiores e clones de Eucalyptus - Revisão aplicada ao melhoramento para produção de pasta celulósica. In: REUNIÃO REGIONAL SOBRE CLONAGEM INTENSIVA EM Eucalyptus, 1., 1994, Aracruz. Anais... Piracicaba: Instituto de Pesquisas Florestais, 1994. p. 1-18.

R. Árvore, Viçosa-MG, v.30, n.5, p.737-747, 2006
FRAMPTON Jr., L. J.; FOSTER, G. S. Field testing vegetative propagules. In: AHUJA, M. R.; LIBBY, W. J. (Eds.) Clonal forestry I, genectics and biotechonology. Springer: Verlag Berlin Heidelberg, 1993. p. 110-134.

GARCIA, S. L. R. Importância de características de crescimento, de qualidade da madeira e da polpa na diversidade genética de clones de Eucalipto. 1998. 103 f. Dissertação (Mestrado em Ciência Florestal) - Universidade Federal de Viçosa, Viçosa, MG, 1998.

INSTITUTO DE PESQUISAS FLORESTAIS - IPEF. REUNIÃO REGIONAL SOBRE CLONAGEM INTENSIVA EM Eucalyptus, 1., 1994, Aracruz. Anais... Piracicaba: IPEF, 1994. 87p.

KIKUTI, P. Parâmetros genéticos em progênies de meios irmãos e clonais numa população de Eucalyptus grandis (Hill) Maiden na região de Telêmaco Borba, PR. 1988. 119 f. Dissertação (Mestrado em Ciência Florestal) - Escola Superior de Agricultura "Luiz de Queiroz", Piracicaba, 1988.

KUREK, A. J. et al. Análise de trilha como critério de seleção indireta para rendimento de grãos em feijão. Revista Brasileira de Agrociência, v. 7. n. 1, p. 29-32, 2001.

LAMBETH, C. et al. Genectic analysis of 16 clonal trials of Eucalyptus grandis and comparisons with seedling checkes. Forest Science, v. 40, n. 3, p. 397-411, 1994.

LEITE, H. G. et al. Descrição e emprego de um modelo para estimar múltiplos volumes de árvores. Revista Árvore, v.19, n.1, p. 75-79, 1995.

LOPES, C. R. Variação fenotípica e genética em clones de Eucalyptus urophylla da ilha das flores (Indonésia). 1992. 110 f. Dissertação (Mestrado em Ciência Florestal) - Escola Superior de Agricultura "Luiz de Queiroz”, Piracicaba, 1992. 
LOPES, M. C. Agrupamento de árvores matrizes de Eucalyptus grandis em função das variáveis dendrométricas e das características tecnológicas da madeira. 2003. 93 f. Dissertação (Mestrado em Ciência Florestal) - Universidade Federal de Santa Maria, Santa Maria, 2003.

Malhotra, N. K. Pesquisa de marketing: uma orientação aplicada. Porto Alegre: Bookman, 2001. 308 p.

MORAIS, O. P. Análise multivariada da divergência genética dos progenitores, índices de seleção e seleção combinada numa população de arroz oriunda de intercruzamento, usando machoesterilidade. 1992. $251 \mathrm{f}$. Tese (Doutorado em Genética e Melhoramento) - Universidade Federal de Viçosa, Viçosa, MG, 1992.

PEREIRA, A. B. Avaliação da eficiência da seleção precoce em famílias de meios irmãos de Eucalyptus camaldulensis Dehnh na região nordeste do estado de Minas Gerais. 1996. 68 f. Dissertação (Mestrado em Genética e Melhoramento de Plantas) -

Universidade Federal de Lavras, Lavras, 1996.
PEREIRA, F. H. F. et al. Divergência genética entre acessos de taro utilizando caracteres morfoqualitativos de inflorescência. Horticultura Brasileira, v. 21, n. 3, p. 520-524, 2003.

REZENDE, G. D. S. P.; BERTOLUCCI, L. G.; RAMALHO, M. A. P. Eficiência da seleção precoce na recomendação de clones de eucaliptos avaliados no norte do Espírito Santo e sul da Bahia. Cerne, v. 1, n. 1, p. 45-50, 1994.

SILVA, R. L. Influência do tamanho da parcela experimental em testes clonais de eucalipto. 2001. 67 f. Dissertação (Mestrado em Ciência Florestal) - Universidade Federal de Viçosa, Viçosa, MG, 2001.

VALENTIN, J. L. Ecologia numérica: uma introdução à análise de dados ecológicos. Rio de Janeiro: Interciência, 2000. 188 p.

XAVIER, A. Silvicultura clonal em Eucalyptus. Revista Madeira, 2003. p. 46-53. (Edição Especial). 